\title{
StudentHistory: um Sistema Web para Análise Visual do Desempenho de Alunos de Graduação
}

\author{
Jonathan Martins Barros Costa, \\ Bruno Santana da Silva, Adja Ferreira de Andrade \\ Instituto Metrópole Digital \\ Universidade Federal do Rio Grande do Norte \\ Av. Senador Salgado Filho, 3000 - 59078-970 - Natal - RN - Brasil \\ jonhmbc@gmail.com, brunodimd.ufrn.br, adja@imd.ufrn.br
}

\begin{abstract}
Student performance analysis at university is difficult, especially due to large number of students and diversity of courses. This work presents the web system StudentHistory for academic performance visual analysis of undergraduates at UFRN. It allows university professors to view Sankey graphs with the flow of students through classes according to their grades. The generation of these graphs occurs automatically, without need of in-depth knowledge of IT, Statistics or Design. This work describes design, development and an evaluation of this tool with participation of educators.
\end{abstract}

Resumo. A análise do desempenho de estudantes de uma universidade é difícil, principalmente pela grande quantidade de alunos e diversidade de cursos. Este trabalho apresenta o sistema web StudentHistory para apoiar a análise visual do desempenho de alunos de graduação da UFRN. Ele permite que docentes da universidade visualizem gráficos Sankey com o fluxo de alunos pelas turmas conforme suas notas. A geração destes gráficos ocorre de forma automática, sem necessidade de conhecimento aprofundado de TI, Estatística ou Design. Este artigo descreve o projeto, o desenvolvimento e uma avaliação desta ferramenta com a participação de pedagogas.

\section{Introdução}

O acompanhamento do desempenho acadêmico de alunos é uma atividade importante para docentes e instituições de ensino. Além de apontar situações em que os alunos demonstram dificuldades, ele permite definir metas de melhoria no ensino e identificar demanda de disciplinas e professores para os próximos períodos. Entretanto, acompanhar o desempenho acadêmico nem sempre é uma tarefa fácil, principalmente quando envolve uma grande quantidade de alunos e uma diversidade de cursos.

A representação visual de dados oferece significativo apoio à cognição humana a partir da percepção do mundo através da visão (Cairo, 2013; Munzner, 2014). Quando feita de forma adequada, bem estruturada, é possível facilitar interpretação de uma grande quantidade de dados em pouco tempo. Nesse sentido, a criação de gráficos e diagramas se torna ferramenta importante para a interpretação e análise de dados sobre o desempenho dos alunos. Aliado a visualização gráfica de dados, temos a evolução e utilização dos sistemas de informação que contribuem principalmente com o poder de processamento computacional. Aproveitar a capacidade de processamento dos 
computadores faz com que análises antes feitas manualmente sejam realizadas cada vez mais rápido e com a menor quantidade de retrabalho humano possível.

Manipulação de uma quantidade significativa de dados, dificuldade em recuperar, organizar e interagir com grande volume de informações, e retrabalho ao construir gráficos semelhantes são exemplos de obstáculos encontrados por docentes e instituições de ensino ao analisar o desempenho acadêmico de alunos universitários. Outro problema importante na análise de desempenho dos estudantes é a falta de acompanhamento contínuo do desempenho dos alunos. Análises feitas esporadicamente ou com dados muito antigos não dão o respaldo que os agentes educacionais necessitam para propor e executar intervenções adequadas. Muitas dessas análises acabam sendo pontuais e não são continuadas devido à dificuldade em manipular e analisar tantos dados com uma frequência semestral ou anual.

O objetivo deste trabalho foi projetar, desenvolver e avaliar o sistema web StudentHistory para apoiar, através da visualização de dados, o acompanhamento do desempenho de alunos de graduação ao longo das disciplinas do curso feito por agentes educacionais integrantes da UFRN (coordenação, colegiado, setor pedagógico, etc.).

\section{Trabalhos Relacionados}

$\mathrm{Na}$ literatura encontramos trabalhos que realizam análises estatísticas sobre dados de desempenho dos alunos em contextos específicos. Por exemplo, Barbosa et al. (2011) analisaram as relações entre indicadores de gestão e desempenho discente, medido pelo ENEM; já Brasil et al. (2018) faz uma revisão sistemática da literatura sobre a análise de aprendizagem em contextos de educação a distância. Outros trabalhos empregam algoritmos de inteligência artificial, data mining e semelhantes para analisar dados sobre o desempenho dos alunos. Couto e Santana (2017), por exemplo, utilizam algoritmos para detecção de padrões na evasão e na retenção em âmbito acadêmico. Cortez e Silva (2008) analisam o desempenho dos alunos nas disciplinas de Matemática e Português no ensino médio usando técnicas de data mining. Por outro lado, a análise visual de dados (Munzner, 2014; Cairo, 2013) ainda é pouco explorada como apoio à compreensão da evolução dos alunos ao longo do curso. Além disso, pouca atenção tem sido dada ao desenvolvimento de ferramentas que auxiliem profissionais de educação analisarem dados sobre o desempenho dos alunos de forma regular e frequente, e não apenas em um esforço pontual.

\section{Projeto do StudentHistory}

O StudentHistory tem como propósito geral apoiar profissionais de educação a analisar o desempenho acadêmico dos alunos de graduação, evitando retrabalho e mantendo o sigilo dos dados. Ele deveria ser de fácil acesso a partir de qualquer máquina com internet, sem necessidade de instalação ou configuração. Os dados deveriam ser os mais atuais possíveis para permitir um acompanhamento contínuo do desempenho. Diante disso, a primeira decisão de projeto foi decidir qual tipo de gráfico ofereceria apoio adequado para visualizar a evolução do desempenho dos estudantes. Ao analisarmos a bibliografia sobre análise visual de dados (Cairo, 2013; Munzner, 2014) e galerias de gráficos disponíveis na web (https://d3js.org, http://datavizcatalogue.com, http://depictdatastudio.com/charts/), optamos por utilizar como base o diagrama Sankey, ilustrado na Figura 1. Ele representa como os integrantes de grupos da esquerda se relacionam com integrantes de grupos da direita, sucessivamente, formando um fluxo de 
informações que vão de um determinado ponto a outro. No contexto do sistema projetado, o fluxo de informações teria como foco principal as notas dos alunos em cada disciplina considerada numa análise.

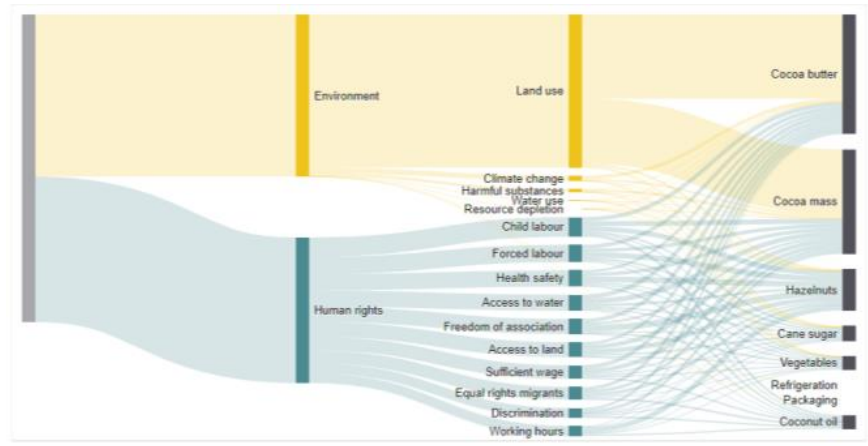

Figura 1. Exemplo de diagrama Sankey.

Fonte: https://bl.ocks.org/wvengen/cab9b01816490edb7083

A Figura 2 mostra o diagrama proposto para o StudentHistory. Cada coluna representa todas as turmas de uma disciplina em determinado semestre, estratificada pelas notas dos alunos nessas turmas: maiores ou iguais a sete em verde escuro; maiores ou iguais a cinco e menores que sete em verde claro; maiores ou iguais a três e menores que cinco em laranja, menores que três em vermelho. Aqueles alunos que não cursaram a disciplina no semestre também seriam indicados em um grupo separado em cinza, totalizando cinco grupos de alunos por disciplina.

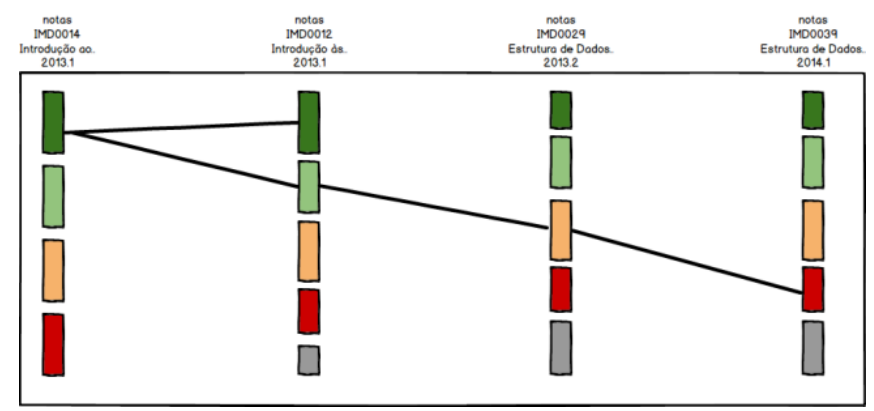

Figura 2. Diagrama Sankey proposto para o StudentHistory.

As ligações entre um grupo da esquerda com outros grupos adjacentes à direita formam um fluxo unidirecional que mostra por quais grupos de notas os alunos passaram nas referidas turmas e semestres. Uma ligação entre dois grupos possui altura proporcional à quantidade de alunos que estão partindo de um grupo de notas e chegando a outro grupo de notas em outra turma, comparada ao total de alunos. O projeto do StudentHistory também levantou um questionamento importante sobre o esforço manual que seria necessário ao docente para a criação de um gráfico como o da Figura 2. $\mathrm{O}$ docente teria que recuperar, organizar e calcular sistematicamente um grande volume de dados. Esse esforço deveria ser melhor empregado na atividade de análise dos dados representados no gráfico, não na sua tabulação e desenho. Assim, o sistema deveria facilitar a criação de gráficos que possibilitem realizar análises específicas. Existem dois atores no StudentHistory: o usuário final, que terá ação em todas funcionalidades do sistema, e a API de Sistemas da Superintendência de Informática (SINFO) da UFRN. As funcionalidades do sistema são: login, logout, listar análises, criar análise, visualizar 
análise, editar análise e excluir análise. A definição do login partiu da necessidade de cada usuário ter acesso apenas às análises que criou. Além disso, o acesso ao sistema deveria ser restrito aos servidores da universidade por envolver dados sigilosos. Inicialmente os usuários previstos são docentes, mas futuramente vão incluir pedagogos e demais profissionais que atuam na assistência pedagógica. Essa restrição de acesso foi decisiva para utilizar a autenticação de usuários da API para realização de login no StudentHistory. Deste modo, garantiríamos que o usuário possua credencial de docente na UFRN e que não seja forçado a lidar com novo login e senha. A Figura 3 ilustra o diagrama de classes (Larman, 2007) do StudentHistory, com a estrutura de classes e relacionamentos, bem como o detalhamento de atributos e métodos para a realização de suas responsabilidades e a colaboração entre classes.

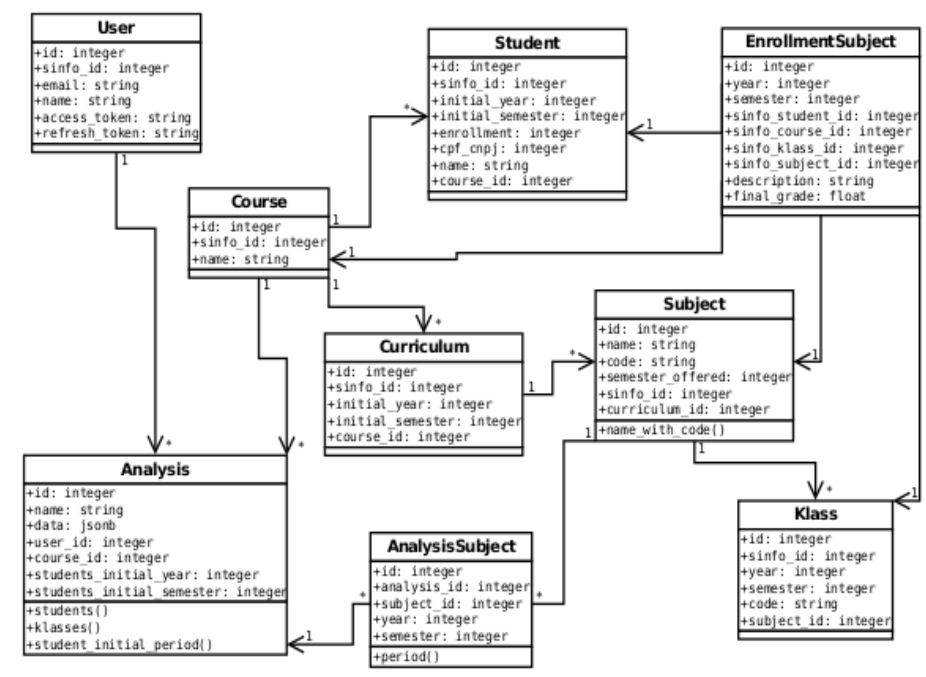

Figura 3. Diagrama de classes do StudentHistory.

A arquitetura do StudentHistory teve como base o padrão MVC, Model-ViewController, (Burbeck, 1987) e foi concebido para a web para atender aos requisitos. Os componentes foram organizados em: parte cliente, parte servidor, banco de dados e API de Sistemas da SINFO. Do lado cliente, partem todas as interações com usuário que fazem requisições http via browser para a parte servidor. A parte cliente também é responsável por renderizar (desenhar) os gráficos, pois no futuro facilitará a evolução do sistema para exibir os mesmos dados de outras formas visando uma compreensão mais rica do contexto educacional. A parte servidor é responsável por receber as requisições do usuário, tratálas e respondê-las, acessando banco de dados e da API.

\section{Desenvolvimento do StudentHistory}

O desenvolvimento do sistema foi de modo incremental e iterativo. Optamos por utilizar a linguagem Ruby e o framework MVC Ruby on Rails pelo padrão arquitetural empregado. A primeira versão do sistema permitia realizar login, logout, listar e criar análises. As análises criadas nesta primeira versão só continham nome e ainda não contavam com a geração do gráfico esperado para sua versão final. O próximo passo foi buscar opções de bibliotecas capazes de gerar o gráfico projetado. Com alguma pesquisa na web1, duas bibliotecas Javascript foram cogitadas por permitirem a criação de 
diagramas Sankey: Google Charts e D3js. Apesar das semelhanças entre essas bibliotecas, D3js foi escolhido pela possibilidade de customização e por oferecer maior controle sobre dados sigilosos.

Tomamos como base o exemplo de d3.chart.sankey (Figura 1). Nesta implementação do diagrama Sankey usando D3js, os nós que possuem apenas ligações partindo deles, ou seja, apenas originam relações, são remanejados totalmente à esquerda; enquanto os nós que são apenas destino de relações são posicionados mais à direita possível no diagrama. Esse comportamento não condiz com o resultado que o StudentHistory gostaria de apresentar por não manter os nós em posições fixas para as turmas e semestres. Portanto, foi necessário alterar a implementação Javascript original para determinar a posição dos nós e rótulos das colunas.

Os dados analisados partiriam da base de dados dos sistemas de gestão da UFRN. Estudamos as informações que precisavam ser recuperadas e como fazer essa recuperação. A consulta dos dados necessários ao StudentHistory foi feita a partir da API (https://api.ufrn.br). A princípio, a ideia era recuperar os dados relacionados aos cursos sob demanda, durante o processo de criação e visualização da análise. Entretanto, foram descobertas algumas limitações técnicas. É necessária uma grande quantidade de requisições à API para recuperar dados dos cursos, alunos, disciplinas, turmas e notas dos alunos para cada análise. Durante a análise do desempenho dos alunos, é muito provável que o usuário faça várias análises similares em seguida para aprofundar e ampliar sua compreensão sobre o histórico dos alunos. Essas análises consecutivas tendem a ser similares, compartilhando curso, disciplinas, turmas e alunos. Se os dados sempre fossem buscados diretamente da API ocorreriam várias requisições repetidas, afetando a eficiência do StudentHistory (por ser mais demorado recuperar da API do que de um repositório local da aplicação) e aumentando significativamente a carga de trabalho do servidor que oferece os serviços da API. Além disso, existem limitações importantes da API que provavelmente foram criadas para evitar sobrecarga no servidor. Cada requisição feita à API retorna no máximo 100 registros por vez. Se a aplicação precisar recuperar mais registros terá que realizar outras requisições utilizando um offset. A API também impõe um limite de 5000 requisições por hora. Estas limitações são significativas para um sistema como o StudentHistory. Por exemplo, a avaliação relatada adiante envolveu aproximadamente 1,5 milhões de registros de dados acadêmicos para apenas três anos de um único curso de graduação.

Por conta dessas limitações da API, uma camada de serviços foi introduzida na arquitetura para importar os dados que serão utilizados nas análises e seus respectivos gráficos. Essa nova camada executa uma série de requisições para a API e salva os resultados em um banco de dados localizado na parte servidor do StudentHistory. Após a importação dos dados, quando o banco de dados já possuía as informações necessárias para a criação de uma análise, houve um novo ciclo no processo de desenvolvimento para implementar as funcionalidades de edição, remoção e visualização das análises (dos gráficos). Também foi alterado o formulário de criação da análise para que o usuário escolha quais informações existentes no banco de dados são relevantes para seu interesse no momento: curso, alunos, turmas, notas, etc. Por fim, como último ciclo na programação do StudentHistory, integramos a biblioteca Javascript D3js para gerar o diagrama Sankey na parte cliente da ferramenta durante a visualização das análises.

A Figura 4 ilustra a página de consulta das análises do usuário, a principal do 
sistema após o login. No canto superior direito é possível identificar o usuário logado e opção para logout. Na parte central encontra-se uma lista das análises que o usuário criou; com seus respectivos nomes, cursos, ano e semestre de ingresso dos estudantes, bem como botões para visualizar, editar ou excluir a análise ou criar uma nova.

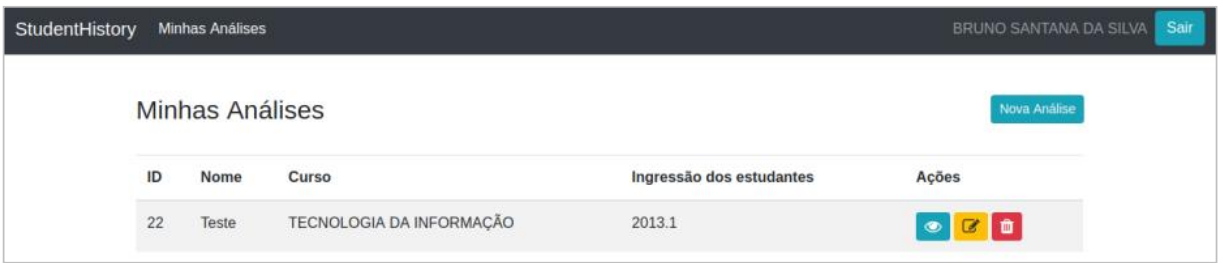

Figura 4. Página de consulta das análises criadas pelo usuário.

A Figura 5 mostra a página de criação de análises. A definição da análise começa com informações básicas como nome da análise, curso a ser analisado, ano e semestre de ingresso dos alunos que serão considerados. Em seguida, a seção de disciplinas permite definir de duas a cinco disciplinas, por código ou nome, selecionar o ano e semestre das turmas ministradas.

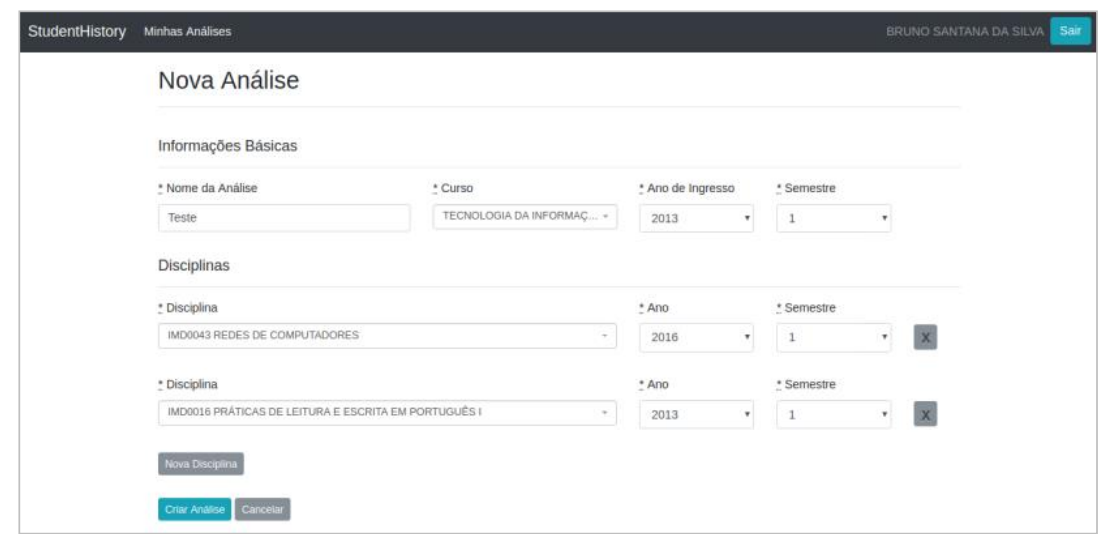

Figura 5. Página de criação de análise.

A Figura 6 apresenta a página de visualização do fluxo de alunos pelas disciplinas conforme seus desempenhos. Nela é possível ver informações básicas: nome, curso, período de ingresso e quantidade total de alunos considerados. A parte central apresenta o diagrama Sankey com indicação das disciplinas, seus códigos, ano e semestre das turmas analisadas. Para cada disciplina e semestre, uma coluna no diagrama foi criada com a presença de cinco grupos de alunos ingressantes conforme suas médias finais naquela disciplina naquele semestre. Os alunos que não se matricularam naquela disciplina no referido semestre formam um grupo à parte. Cada ligação entre as colunas indica que um grupo de alunos que obteve determinada média final numa disciplina à esquerda obteve determinada média final em outra disciplina à direita, ou não se matriculou nelas. A largura das linhas entre colunas indica a quantidade de alunos naquela situação, proporcional ao total de ingressantes. 


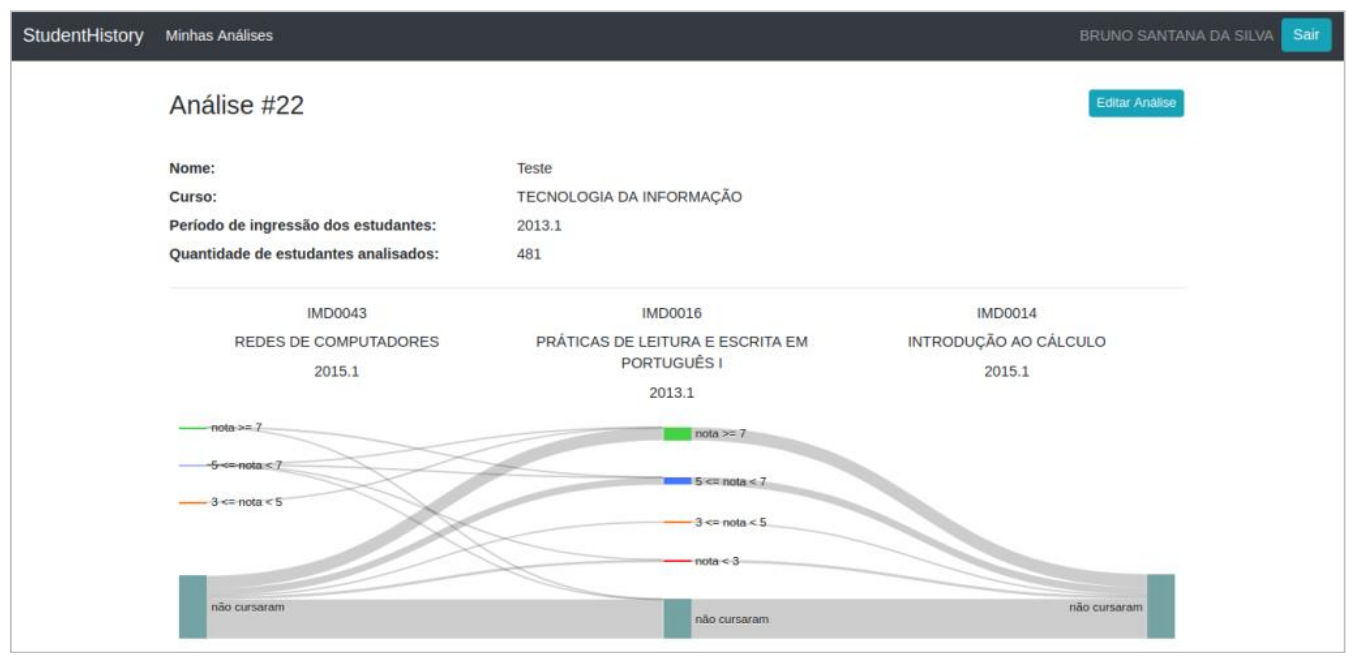

Figura 6. Página de visualização da análise com dados de teste.

\section{Avaliação do StudentHistory}

Avaliamos o StudentHistory considerando os seguintes objetivos: (1) verificar se os participantes conseguem criar análises e o tempo necessário para a execução das tarefas propostas; (2) comparar a compreensão dos diagramas pelos participantes e pelos desenvolvedores do sistema; e (3) consultar a opinião dos participantes sobre o uso da ferramenta e sugestões de melhorias.

As metodologias utilizadas nesta avaliação foram observação de uso e grupo focal (Barbosa e Silva, 2010). Três pedagogas do Setor Pedagógico do IMD aceitaram participar desta pesquisa após esclarecimentos, conforme assinatura de termo de consentimento. Elas realizaram individualmente duas tarefas usando o StudentHistory. $\mathrm{Na}$ Tarefa 1, elas criaram uma análise contendo cinco disciplinas de matemática ou programação do curso de BTI da UFRN. Na Tarefa 2, elas interpretaram o gráfico de uma análise já existente no sistema (Figura 7).

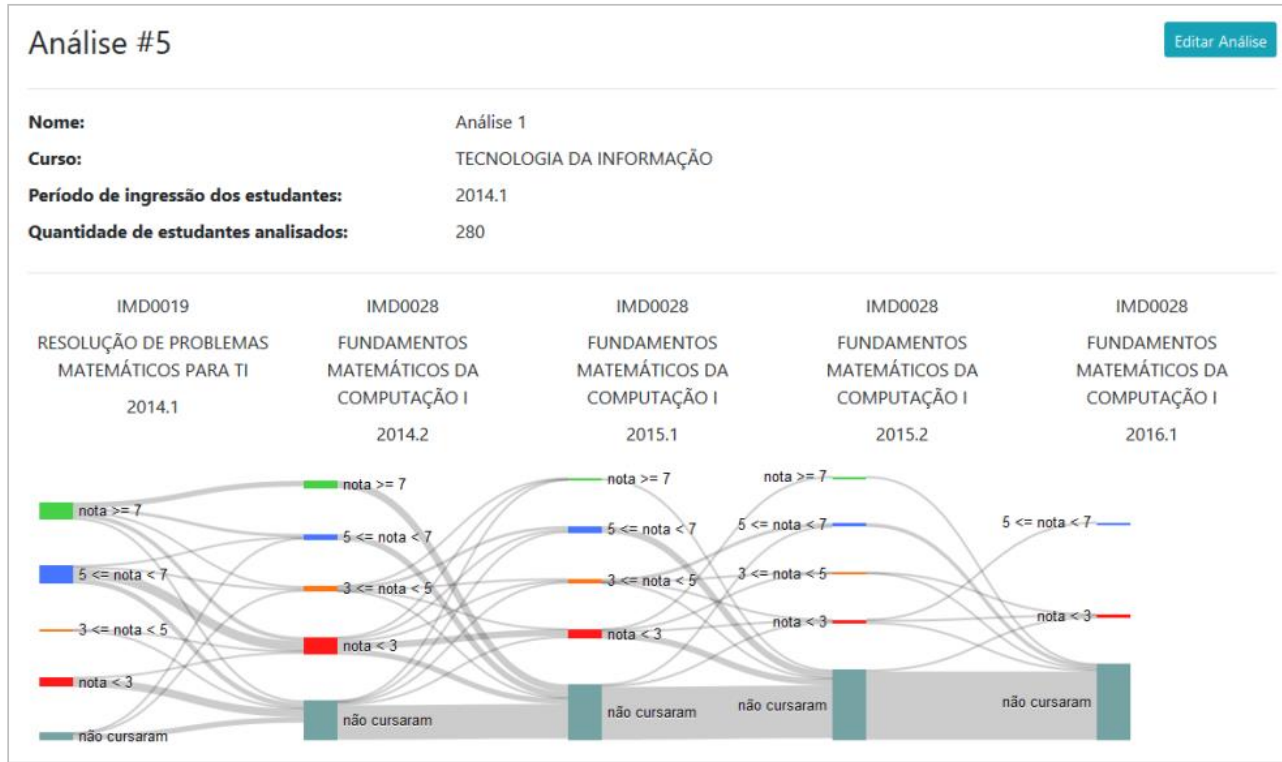

Figura 7. Página de visualização da análise com dados reais. 
As execuções destas tarefas foram observadas e gravadas em vídeo. As participantes escreveram observações sobre o desempenho dos alunos analisados, conforme sua compreensão. Após a realização das tarefas, as participantes realizaram uma discussão em grupo a respeito da sua opinião sobre o StudentHistory, o potencial da ferramenta na prática profissional e sugestões de melhoria. Essa discussão foi registrada em áudio. Os vídeos de interação foram analisados para contabilizar o tempo de realização e o sucesso da conclusão das tarefas. As observações escritas pelos participantes foram analisadas para determinar compatibilidade com a intenção dos desenvolvedores. $\mathrm{O}$ áudio das discussões do grupo focal foi analisado para identificar recorrências nas opiniões dos participantes, bem como outros aspectos relevantes.

\subsection{Resultados da observação de uso}

Na Tarefa 1, P1 (Participante 1) levou 8 minutos para criar a análise. Grande parte desse tempo foi gasto com a visualização das disciplinas disponíveis. Apesar da conclusão parcial da tarefa de criar uma análise, P1 não ficou satisfeito com o gráfico resultante, pois o conjunto de alunos analisados e o período das disciplinas escolhidas não geraram um gráfico com variações de notas suficientes para a observação do desempenho dos alunos. P1 editou a análise quatro vezes e mudou ano e semestre de ingresso dos alunos, disciplinas, ano e semestre das disciplinas, em busca de um gráfico com mais ligações entre os grupos de notas. P1 concluiu que a falta de conhecimento do currículo e da oferta das disciplinas do curso dificultou na escolha de disciplinas que pudessem gerar um gráfico mais interessante.

P2 demorou aproximadamente 8 minutos para realizar a criação da primeira análise. Ao longo da Tarefa 1, P2 observou uma análise já criada no sistema, tentou salvar a análise com campos obrigatórios em branco e os corrigiu após validação do sistema. Criou outras duas análises extras. Em uma das análises criadas por P2, foi selecionado o período 2017.2 para ingresso dos alunos e nenhum gráfico foi gerado, pois o curso de BTI da UFRN não possui alunos ingressantes para esse semestre. Em suas observações, P2 escreveu algumas sugestões como botões de navegação para voltar entre telas e o sistema apresentar como opções apenas o que foi ofertado.

P3 levou pouco menos de 7 minutos para concluir a criação da primeira análise durante a Tarefa 1. Ela editou quatro vezes a análise criada. Na primeira edição, alterou $\mathrm{o}$ ano de ingresso dos estudantes. Na segunda e terceira edição, P3 removeu uma das disciplinas selecionadas e inseriu outra disciplina. Na última edição feita, ela editou o semestre das turmas analisadas de uma das disciplinas. P3 relatou que o gráfico fica mais claro ao longo do uso e, assim como P1, constatou a necessidade do conhecimento da grade curricular do curso analisado. P3 sugeriu que o sistema alertasse os usuários caso escolhessem uma disciplina que ainda não foi cursada pelo grupo de alunos analisados. P3 teve dificuldades em compreender a rótulo do link entre disciplinas que informa quantos alunos saem e chegam em grupos de alunos que "não cursaram". Na Tarefa 2, os participantes compreenderam o universo de estudantes ingressantes analisados $\mathrm{e}$ conseguiram identificar que uma coluna representava alunos agrupados por nota ou sem matrícula em determinada disciplina. Apesar disso, houve relato sobre a falta de alinhamento entre o gráfico e os elementos que informam as disciplinas presentes na análise. P1 tomou nota sobre os componentes curriculares analisados e o desempenho de grupos de alunos em determinados semestres e considerou as disciplinas escolhidas nesta tarefa como críticas pelo alto número de reprovações e desistências. Relatou que entre 
uma disciplina e outra existiram comportamentos diferenciados. Em alguns casos alunos aumentaram as notas, enquanto em outros obtiveram rendimento inferior, inclusive nos casos de repetência em um semestre seguinte para uma mesma disciplina. P2 levantou questionamentos se a espessura dos links está relacionada com a evolução do desempenho. Também menciona sobre o rótulo informar a quantidade de alunos em cada grupo presente no gráfico. P3 relata sobre a relação das notas entre as disciplinas analisadas e a taxa de aprovação e retenção de alunos em uma mesma disciplina.

\subsection{Resultados do Grupo Focal}

P3 achou o sistema interessante por dar uma noção visual do que aconteceu com um grupo de alunos do curso, diferente do histórico escolar individual padrão que apresenta toda a vida acadêmica de apenas um aluno por vez. No histórico em formato de tabela, ela relatou dificuldade em comparar o desempenho de um aluno com um conjunto de outros alunos do curso e em analisar sua evolução ao longo do tempo, principalmente quando se trata de reprovações. P1 falou sobre a possibilidade de analisar a evolução do desempenho dos alunos em um componente curricular ao longo do tempo, o índice de desistência de cursar a disciplina e o índice de sucesso para os casos de repetição de uma disciplina. P1 ficou fascinado com as possibilidades que o sistema lhe oferece. P2 comenta sobre o sistema possibilitar a análise do desempenho de alunos em diferentes disciplinas prérequisito/co-requisito de outras disciplinas e permitir questionamentos do tipo "na disciplina A ele (o aluno) foi bem sucedido e na disciplina B não. Por quê?’. P2 também comenta sobre a possibilidade de avançar nos semestres para uma mesma disciplina a fim de uma análise com visão voltada a outros fatores além dos alunos, como por exemplo, troca de docentes que ministram as turmas da disciplina ou até mesmo tentar relacionar fatores externos que possam ter influenciado o desempenho dos alunos, como por exemplo, greves na universidade, protestos e paralisações na cidade.

Sobre o potencial de uso no trabalho dos pedagogos, P2 cita a possibilidade de expansão da ferramenta para outros vínculos da UFRN (técnicos administrativos) e adaptações na utilização do sistema com foco na avaliação de cursos em outros níveis de ensino na universidade, como básico e técnico. Também foi levantamento o questionamento do uso do sistema para avaliar o desempenho acadêmico durante o semestre corrente na tentativa de tentar identificar e corrigir problemas no início, e não apenas para os semestres concluídos. P1 relata sobre a dificuldade em realizar análises de desempenho manualmente para uma grande quantidade de alunos pelo esforço e tempo necessários. Isso inviabiliza a realização de um acompanhamento contínuo dos alunos durante o andamento dos cursos, o que atrapalha a tomada de providências necessárias, como, por exemplo, encaminhamento para o plantão pedagógico, apoio de monitoria para reforço e esclarecimento de dúvidas. Os participantes consideraram que a visualização e compreensão do fluxo de alunos pelas disciplinas permite avaliar disciplinas com alto índice de reprovação, tentar entender os motivos das desistências (abandono de uma turma em determinado semestre) e o que acontece com os alunos a ponto de não haver uma melhora nas notas ao longo das reprovações em disciplinas. A respeito das sugestões de melhoria, P1 diz que seria interessante indicação das disciplinas com dados disponíveis conforme ano de ingresso dos alunos selecionados, a fim de evitar análises que gerem gráficos com poucos estudantes que cursaram as disciplinas. P2 aconselhou sobre a existência de legenda para sinalizar disciplinas que são pré-requisito de outras ou que não foram ofertadas no período escolhido. P1, P2 e P3 também sugerem a possibilidade de 
analisar as turmas das disciplinas durante o decorrer do semestre, com a separação das notas por unidades, com o objetivo de tentar antecipar reprovações e como ferramenta de autoavaliação dos docentes sobre suas turmas durante o semestre em execução. Também foi apontada a necessidade de analisar as turmas considerando os professores e a indicação de um grupo de alunos aprovados distinto do grupo de alunos que "não cursaram" a disciplina. Apesar das dificuldades inicialmente demonstradas, os participantes obtiveram uma compreensão do sistema e dos gráficos próxima da esperada pelos desenvolvedores. Identificaram os elementos gráficos dos diagramas, o universo de alunos ingressantes, as disciplinas analisadas e relacionaram as ligações entre os grupos de cada disciplina como parte do fluxo de desempenho desses alunos ao longo do curso. Para realização de análises interessantes, é importante ter um bom conhecimento acerca do currículo do curso, bem como ter acesso a um tutorial sobre o uso do gráfico Sankey.

\section{Considerações Finais}

Este trabalho apresentou o StudentHistory, uma aplicação web que busca auxiliar docentes da UFRN na análise do desempenho dos estudantes de graduação. O sistema apresenta um diagrama do tipo Sankey com o fluxo de estudantes entre as disciplinas analisadas de acordo com suas respectivas notas. O sistema conta com integração à API de Sistemas da SINFO, através da qual são recuperados dados utilizados nas análises, além de permitir autenticação com as credenciais de acesso já existentes no SIGAA. Trabalhos futuros podem aprofundar a avaliação do sistema considerando a usabilidade e comunicabilidade para a diversidade de profissionais de educação envolvidos, implementar melhorias na ferramenta, e explorar novas visualizações de informação sobre o desempenho dos alunos.

\section{Referências}

Barbosa, G.C.; Freire, F.S.; Crisostomo, V.L. (2011) Análise dos indicadores de gestão das IFES e o desempenho discente no ENADE. Avaliação, v. 16, n. 2, p. 317-344.

Barbosa, S.D.J.; Silva, B.S. (2010) Interação Humano-Computador. Elsevier.

Cairo, A. (2013) The Functional Art. Berkeley, CA: New Riders.

Couto, D.C.; Santana, A.L. (2017) Mineração de Dados Educacionais Aplicada à Identificação de Variáveis Associadas à Evasão e Retenção. Anais do II Congresso sobre Tecnologias na Educação (Ctrl+e 2017). Rio Tinto, Paraíba, p. 333-344.

Cortez, P.; Silva, A. (2008). Using data mining to predict secondary school student performance. In Proceedings of 5th Annual Future Business Technology Conference, Porto, Portugal, pages 5-12.

Larman, C. (2007) Utilizando UML e padrões: uma introdução à análise e ao projeto orientados a objetos e ao desenvolvimento interativo. 3.ed. Porto Alegre: Bookman.

Munzner, T. (2014) Visualization Analysis and Design. AK Peters.

Brasil, P.C.; Medeiros, T.J.; Nunes, I.D. (2018) Uma Revisão Sistemática Sobre o Uso de Learning Analytics em Ambientes Virtuais de Aprendizagem Brasileiros. Anais do III Congresso sobre Tecnologias na Educação (Ctrl+e 2018). Fortaleza, p. 371-380. 